\title{
MODERNIZAÇÃO DA AGRICULTURA NOS MUNICÍPIOS DO NORDESTE PARAENSE: DETERMINANTES E HIERARQUIZAÇÃO NO ANO DE 2006 ${ }^{1}$
}

\author{
Fabrício Khoury Rebello ${ }^{2}$ \\ Marcos Antônio Souza dos Santos ${ }^{3}$ \\ Alfredo Kingo Oyama Homma
}

\begin{abstract}
Resumo: Este artigo analisa o nível de modernização agrícola da mesorregião do Nordeste Paraense, no estado do Pará, para o ano de 2006, bem como avalia as mudanças ocorridas em relação à década anterior. Para descrever o padrão tecnológico e a classificação relativa dos municípios, utilizou-se a técnica de análise fatorial para construção de um índice de modernização agrícola. Os resultados mostram a existência de certa discrepância no nível tecnológico entre os municípios analisados, ainda que se tenha percebido uma evolução positiva entre os dois períodos avaliados. Dos 49 municípios estudados, dez atingiram índices de modernização acima de 50\% (nível tecnológico 1, o mais elevado). São eles: Capitão Poço, Curuçá, Igarapé-Açu, Moju, Peixe-Boi, Santarém Novo, São João da Ponta, São Miguel do Guamá, Tailândia e Tomé-Açu. Por sua vez, no nível tecnológico 3, o mais atrasado, encontram-se 17 municípios, com destaque para São Domingos do Capim, Cametá, Oeiras do Pará, Colares e Limoeiro do Ajuru, que têm as piores posições no ranking. Estudos dessa natureza são fundamentais para orientar políticas públicas em prol do desenvolvimento rural sustentável na Amazônia, particularmente no Nordeste Paraense.
\end{abstract}

Palavras-chave: Análise Fatorial. Desenvolvimento Rural - Estado do Pará. Meio Ambiente.

\footnotetext{
Recebido em: 25/04/2011. Aceito em: 15/08/2011.

2 Doutorando em Ciências Agrárias, área de concentração em Agroecossistemas da Amazônia(UFRA/EMBRAPA Amazônia Oriental). E-mail: fabriciorebello@unama.br// fabriciorebello@hotmail.com

3 Professor do Instituto Socioambiental e dos Recursos Hídricos (ISARH) da Universidade Federal Rural da Amazônia (UFRA).E-mail: marcos.santos@ufra.edu.br

4 Professor Visitante da Universidade Federal Rural da Amazônia e pesquisador da Empresa Brasileira de Pesquisa Agropecuária.E-mail: homma@cpatu.embrapa.br
} 
Abstract: This paper analysis the level of agricultural modernization in the Northeast region of Pará State in 2006, as well as changes occurred in relation to the previous decade. To describe the technological standard and the relative ranking of municipalities, we used the factor analyzes statistical method to construct a ratio of agricultural modernization. The results showed the existence of a large discrepancy in technological level between the municipalities analyzed,. Of the 49 municipalities examined, only ten have reached the level of modernization above $50 \%$ (the highest level is 1 ). They are: Capitão Poço, Curuçá, Igarapé-Açu, Moju, Peixe-Boi, Santarém Novo, São João da Ponta, São Miguel do Guamá, Tailândia, and Tomé-Açu. On the other hand, 17 municipalities expressed technological level of 3, the lowest one. They are: São Domingos do Capim, Cametá, Oeiras do Pará, Colares and Limoeiro do Ajuru with the worst rankings. These studies are fundamental to orient public policies focusing on sustainable rural development in the Amazon, especially in the Northeast of Pará.

Keywords: Factor Analysis. Rural Development - State of Pará. Environment.

\section{Introdução}

O processo de modernização da agricultura brasileira tem início na década de 1950 com a importação de meios de produção mais avançados. É na década de 1960, no entanto, com a implantação da indústria de equipamentos e insumos no Brasil que esse processo vai se estabelecer concretamente. $\mathrm{O}$ crédito subsidiado, principalmente para a compra de insumos modernos e financiamento de capital, a extensão rural e a pesquisa agropecuária foram instrumentos de política essenciais para alavancar patamares mais elevados de modernização no setor rural nacional.

Ainda que se percebam avanços no processo de modernização da agricultura no País, muitas regiões evoluem mais lentamente, o que de certa forma acaba por pressionar a base de seus recursos naturais, percebidas, por exemplo, nas altas taxas de desmatamento e queimadas, erosão do solo, degradação dos recursos hídricos e na perda de biodiversidade. O estado do Pará e a mesorregião do Nordeste Paraense, em particular, são exemplos desse avanço vagaroso. 
Fabrício Khoury Rebello, Marcos Antônio Souza dos Santos \& Alfredo Kingo Oyama Homma

Alguns dados dão a dimensão dessa defasagem tecnológica. Enquanto no estado do Pará, $27,02 \%$ dos estabelecimentos agrícolas têm acesso à energia elétrica, insumo estratégico no processo de modernização, no conjunto do País, esse percentual é de $68,14 \%$, e no estado de São Paulo, atinge o patamar de $81,43 \%$ dos estabelecimentos rurais (IBGE, 2010). Conhecer o estágio de desenvolvimento do setor rural é fundamental, portanto, para estabelecer políticas públicas consistentes com vistas a reduzir esse distanciamento tecnológico.

No caso da Amazônia, em especial, a indução do processo de modernização agrícola assume proporções contundentes conforme apontado por Rebello e Homma (2009). Nessa linha, o presente estudo tem por objetivo apresentar um diagnóstico do nível de modernização da agricultura dos municípios da mesorregião do Nordeste Paraense, estimando o índice de modernização agrícola e seus fatores determinantes para o ano de 2006, bem como efetuar uma comparação entre os níveis tecnológicos em dois momentos distintos no tempo - os anos de 1995/ 1996 e 2006. O estudo se justifica por se tratar de uma região de fronteira onde o avanço da agropecuária terá que lidar com questões tecnológicas e ambientais, sendo importante o conhecimento da realidade para o delineamento de políticas de desenvolvimento.

\section{Marco teórico}

Um dos principais problemas tecnológicos enfrentados na Amazônia diz respeito ao uso do sistema de derruba e queima de floresta densa e/ou vegetação secundária, principalmente pelos pequenos produtores. Teoricamente, esse processo está associado à abundância de terra e à limitação de mão de obra e vice-versa, características distintas existentes na Amazônia (HOMMA, 1998). O enfrentamento desta situação tornase, portanto, relevante para equacionar a questão ecológica dos desmatamentos e queimadas versus o aspecto da racionalidade econômica. 
Na Amazônia, a adoção de tecnologias modernas assume preços relativos bastante elevados quando comparados com outras regiões do País, como também é mais onerosa que a incorporação de novas áreas da fronteira agrícola pelo processo tradicional. Desta forma, a melhoria do padrão tecnológico na região passa, pois, pelo crescimento da agricultura a partir da intensificação do uso da terra em áreas já ocupadas, conforme o modelo prescrito por Penna e Mueller (1977). Deve-se, ainda, considerar as circunstâncias em que a modernização e o dualismo tecnológico estão presentes, conforme preconizado por Paiva (1975). Isso, certamente, passa pela premissa de uma maior racionalização e coordenação no planejamento do desenvolvimento regional.

\section{Metodologia}

\section{1. Área de estudo}

A mesorregião do Nordeste Paraense é formada por cinco microrregiões: Bragantina, Cametá, Guamá, Salgado e Tomé-Açu e por 49 municípios. Ocupa uma superfície correspondente a $10,6 \%$ da área do estado do Pará (135.000 km²) e um contingente populacional de 1,6 milhão de habitantes, equivalentes a cerca de $27 \%$ da população paraense (IBGE, 2009). O Mapa 1 apresenta a delimitação geográfica da área de estudo e a relação dos municípios pode ser visualizada na Tabela 3. 


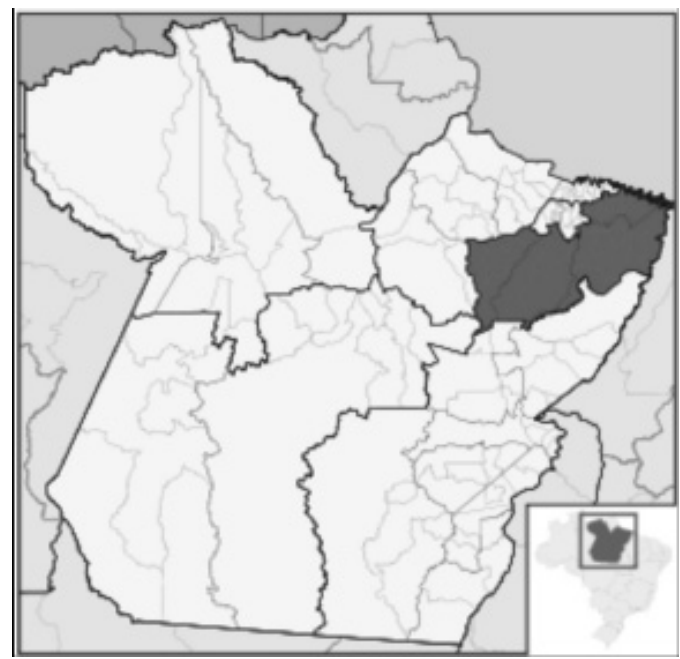

Mapa 1 - Delimitação geográfica da mesorregião do Nordeste Paraense, estado do Pará.

Essa área representa um dos maiores polos de produção agropecuária da economia paraense, respondendo, no ano de 2008 , por $42,09 \%$ do Valor Bruto da Produção (VBP) das culturas permanentes (o Sudoeste Paraense, segunda maior participação, respondeu com $28,46 \%$ do VBP). Quanto às culturas temporárias e a pecuária, no mesmo período, participou com $26,21 \%$ e $9,19 \%$ do VBP, respectivamente (IBGE, 2010a).

O Nordeste Paraense é uma das mais antigas áreas de colonização agrícola da Amazônia, com o processo tendo se iniciado por volta de 1875 (EGLER, 1961; PENTEADO, 1967). Historicamente, o crescimento de sua população resultou da migração provocada pela construção da Estrada de Ferro de Bragança (1883-1908) e pelo boom da borracha (1879-1912). A consequência lógica desse crescimento populacional foi o desenvolvimento das cidades, da rede rodoviária e dos diversos tipos de produção agrícola que levaram à sua intensa antropização. As áreas de floresta primária, que há um século, ainda, cobriam quase totalmente a região, praticamente desapareceram e correspondem a menos de 5\% da superfície total das propriedades agrícolas familiares (BILLOT, 1995). 
A área de estudo apresenta, portanto, significativa importância dentro do contexto econômico e histórico da dinâmica produtiva da Amazônia, sendo uma relevante referência para a formulação de políticas públicas. A seguir, será apresentado o modelo de análise utilizado neste estudo.

\subsection{Análise fatorial}

A análise fatorial é uma técnica estatística multivariada empregada na redução e sumarização de dados. Permite analisar as relações entre um amplo conjunto de variáveis correlacionadas, simplificando-as por meio da definição de um conjunto de dimensões latentes comuns, denominadas de fatores (HAIR et al., 2006; MANLY, 2008; MINGOTI, 2005).

O modelo básico de análise fatorial pode ser especificado pela seguinte expressão:

$$
X_{i}=A_{i 1} F_{1}+A_{i 2} F_{2}+A_{i 3} F_{3}+\ldots+A_{i k} F_{k}+U_{i}+E_{i}
$$

Em que:

$X_{i}=$ são as variáveis que especificam os indicadores de modernização;

$F_{k}=$ são os k-ésimos fatores comuns;

$A_{i k}=$ são as cargas fatoriais que indicam a intensidade das relações entre as variáveis $X_{i}$ e os fatores;

$U_{i}=$ é o fator único que especifica a parte da variância total que não se associa com a variância de outras variáveis; e

$E_{i}=$ fator de erro que representa o erro de observação, de mensuração ou de especificação do modelo. 
Fabrício Khoury Rebello, Marcos Antônio Souza dos Santos \& Alfredo Kingo Oyama Homma

A aplicação da análise fatorial pressupõe a existência de correlação entre as variáveis. Para aferir a qualidade dessas correlações de forma a prosseguir com a análise, foram utilizados os testes de esfericidade de Bartlett e de Kaiser-Meyer-Olkin (KMO). O primeiro testa a hipótese nula de a matriz de correlações ser uma matriz Identidade, cujo determinante é igual a um. O segundo, cujo valor varia entre zero e um, tem a finalidade de comparar as correlações de ordem zero com as correlações parciais observadas entre as variáveis (MINGOTI, 2005).

A partir da utilização do software SPSS (versão 17.0), foram estimados os fatores pelo método de componentes principais e selecionados aqueles que apresentaram raízes características superiores à unidade. Com base nos fatores extraídos, foram estimados os escores fatoriais para cada um dos municípios do Nordeste Paraense. A estimação dos escores foi realizada por meio do método semelhante ao da regressão. A expressão geral para estimação do j-ésimo escore fatorial $\left(F_{j}\right)$ é dada por:

$$
F_{j}=W_{i 1} X_{1}+W_{i 2} X_{2}+W_{i 3} X_{3}+\ldots+W_{i p} X_{p}
$$

Em que $W_{j i}$ são os coeficientes dos escores fatoriais e $p$ é o número de variáveis.

\subsection{Construção do Índice de modernização agrícola}

A hierarquização da modernização agrícola dos municípios do Nordeste Paraense foi realizada a partir dos escores fatoriais, ou seja, dos valores dos fatores para cada uma das 49 observações (municípios). A partir da expressão 3, obteve-se um Índice Bruto de Modernização Agrícola $(I B M A)$ por meio do cálculo da média dos fatores ponderada pela proporção de explicação da variância total associada a cada um deles. Aplicações como esta podem ser encontradas em diversos trabalhos como os desenvolvidos por Cunha, Lima e Moura (2005); Cunha et al. (2008) e Melo e Parré (2006). 
$I_{i}=\frac{\sum_{j=1}^{3} w_{j} \cdot F_{j i}}{\sum_{j=1}^{3} w_{j}}$

Em que:

$I B M A_{i}=$ é o Índice Bruto de Modernização Agrícola do i-ésimo município;

$w_{i}=$ proporção da variância explicada por cada fator; e

$F_{j i}=$ j-ésimo escore fatorial associado a cada um dos municípios.

Visando a estabelecer a ordenação dos municípios, segundo o nível de modernização, foi então determinado o Índice de Modernização Agrícola (IMA) para cada um deles. O IMA foi obtido pela padronização do IBMA, procedimento que permite que os valores variem no intervalo de zero a 100, conforme a expressão:

$$
I M A_{i}=\left(\frac{I B M A_{i}-I B M A^{\min }}{I B M A^{\max }-I B M A^{\min }}\right) \cdot 100
$$

Em que:

$I B M A^{\text {min }}=$ é o menor $I B M A$ observado entre todos os municípios; e

$I B M A^{\text {max }}=$ é o maior $I B M A$ observado entre todos os municípios.

A partir dos valores do IMA, foram estabelecidos três níveis tecnológicos, conforme especificado abaixo: 
a) $I M A_{i} \geq 50$ = nível tecnológico 1 ;

b) $25<I M A_{i}<50=$ nível tecnológico 2; e

c) $0<I M A_{i}<25$ = nível tecnológico 3.

\subsection{Definição das variáveis}

Os dados utilizados no trabalho foram obtidos do Censo Agropecuário de 1995/1996 e 2006 do IBGE (1998; 2010). Foram construídos 17 indicadores para refletir o processo de modernização agrícola dos municípios do Nordeste Paraense $\left(X_{1}\right.$ a $\left.X_{17}\right)$ para o ano de 2006, conforme especificado no Quadro 1.

Quadro 1 - Definição dos indicadores para análise fatorial.

\begin{tabular}{|c|c|}
\hline Indicadores & Especificação \\
\hline $\mathrm{X}_{1}$ & $\mathrm{n}^{\circ}$ de estabelecimentos com acessoà energia elétrica (\%); \\
\hline $\mathrm{X}_{2}$ & $\mathrm{n}^{\circ}$ de estabelecimentos com indicação de uso de adubação (\%) \\
\hline $\mathrm{X}_{3}$ & $\mathrm{n}^{\circ}$ de estabelecimentos com adoção de práticas agríœlas $(\%)$ \\
\hline $\mathrm{X}_{4}$ & $\mathrm{n}^{\circ}$ de estabelecimentos com acesso aos serviços deAssist. Téc. e Extensão Rural (Ater)(\%); \\
\hline $\mathrm{X}_{5}$ & $\mathrm{n}^{\circ}$ de estabelecimentos com indicação de uso de agrquímicos $(\%)$; \\
\hline $\mathrm{X}_{6}$ & $\mathrm{n}^{\circ}$ de estabelecimentos com indicação de usos de irrigação (\%) \\
\hline $\mathrm{X}_{7}$ & $\mathrm{n}^{\circ}$ de estabelecimentos com indicação de uso de trator (\%) \\
\hline $\mathrm{X}_{8}$ & $\mathrm{n}^{\circ}$ de estabelecimentos com indicação de acesso a financiamento (\%) \\
\hline $\mathrm{X}_{9}$ & valor do financiamento em relação ao $\mathrm{n}^{\circ}$ deestabelecimentos ( $\mathrm{R} \$ /$ estabelecimento); \\
\hline $\mathrm{X}_{10}$ & valor dos investimentos em relação ao $\mathrm{n}^{\circ}$ deestabelecimentos (R $\$$ /estabelecimento) \\
\hline $\mathrm{X}_{11}$ & $\begin{array}{l}\text { valor das despesas com adubos, corretivos, sementes e mudas em relação ao } \mathrm{n}^{\circ} \mathrm{de} \\
\text { estabelecimentos (R } \$ \text { /estabelecimento) }\end{array}$ \\
\hline $\mathrm{X}_{12}$ & valor das despesas totais em relação ao pessoal ocupado nos estabelecimentos (R\$/pessoa) \\
\hline $\mathrm{X}_{13}$ & valor bruto da produção por estabelecimento (R\$/estabelecimento) \\
\hline $\mathrm{X}_{14}$ & valor bruto da produção por pessoal ocupado (R\$/pessoa ocupada) \\
\hline $\mathrm{X}_{15}$ & $\begin{array}{l}\text { valor das despesas com agroquímicos em relação ao } \mathrm{n}^{\circ} \text { de estabelecimentos } \\
\text { (R\$/estabelecimento); }\end{array}$ \\
\hline $\mathrm{X}_{16}$ & $\begin{array}{l}\text { valor das despesas com energia elétrica e combustíveis em relação ao } \mathrm{n}^{\circ} \text { de estabelecimentos } \\
\text { (R\$/estabelecimento); }\end{array}$ \\
\hline $\mathrm{X}_{17}$ & valor das despesas totais em relação ao $\mathrm{n}^{\circ}$ de estabelecimentos ( $\mathrm{R} \$$ /estabelecimento) \\
\hline
\end{tabular}

Fonte: Dados da pesquisa. 


\section{Resultados e discussão}

\subsection{Fatores determinantes e hierarquização da modernização da agricultura em 2006}

A aplicação da análise fatorial proporcionou a extração de quatro fatores com raízes características superiores à unidade, as quais sintetizam as informações contidas nas 17 variáveis originais. Estes fatores explicam $71,634 \%$ da variância total do modelo (Tabela 1).

O teste de Bartlett foi significativo a níveis inferiores a $1 \%$ de probabilidade, rejeitando a hipótese nula de que a matriz de correlação seja uma matriz Identidade. O teste de Kaiser-Meyer-Olkin (KMO) apresentou um valor de 0,639 , indicando que o método é adequado à análise dos dados (Tabela 1).

Tabela 1 - Raiz característica e percentual da variância explicada por cada fator.

\begin{tabular}{c|c|c|c}
\hline Fatores & Raízes Características & Variância Explicada pelo Fator (\%) & Variância Acumulada (\%) \\
\hline F1 & 5,167 & 30,395 & 30,395 \\
F2 & 2,697 & 15,862 & 46,258 \\
F3 & 2,382 & 14,012 & 60,269 \\
F4 & 1,932 & 11,364 & 71,634 \\
\hline
\end{tabular}

Fonte: Dados da pesquisa.

Nota: Teste de esfericidade de Bartlett $=760,281(\mathrm{p}<0,01)$ e $\mathrm{KMO}=0,639$.

A Tabela 2 mostra as cargas fatoriais e as comunalidades para os quatro fatores considerados. Os valores obtidos para a comunalidade revelam que, praticamente, todas as variáveis têm sua variabilidade, significativamente, captada e representada pelos quatro fatores. 
Fabrício Khoury Rebello, Marcos Antônio Souza dos Santos \& Alfredo Kingo Oyama Homma

Tabela 2 - Cargas fatoriais após rotação ortogonal e as respectivas comunalidades.

\begin{tabular}{|c|c|c|c|c|c|}
\hline \multirow{2}{*}{ Indicadores } & \multicolumn{4}{|c|}{ Fatores } & \multirow{2}{*}{ Comunalidades* } \\
\hline & F1 & $\mathrm{F} 2$ & F3 & $\mathrm{F} 4$ & \\
\hline$\overline{X_{1}}$ & 0,08 & 0,79 & $-0,21$ & $-0,05$ & 0,675 \\
\hline $\mathrm{X}_{2}$ & 0,07 & $\mathbf{0 , 8 0}$ & $-0,04$ & 0,38 & 0,789 \\
\hline $\mathrm{X}_{3}$ & $-0,17$ & $\mathbf{0 , 5 3}$ & 0,16 & $-0,27$ & 0,407 \\
\hline $\mathrm{X}_{4}$ & 0,34 & 0,09 & $-0,08$ & 0,71 & 0,631 \\
\hline $\mathrm{X}_{5}$ & 0,10 & 0,72 & 0,27 & 0,44 & 0,797 \\
\hline $\mathrm{X}_{6}$ & 0,05 & 0,66 & 0,02 & 0,08 & 0,448 \\
\hline $\mathrm{X}_{7}$ & 0,79 & 0,17 & 0,08 & 0,41 & 0,827 \\
\hline $\mathrm{X}_{8}$ & $-0,01$ & 0,09 & 0,02 & 0,84 & 0,720 \\
\hline $\mathrm{X}_{9}$ & 0,23 & $-0,24$ & 0,57 & 0,08 & 0,441 \\
\hline $\mathrm{X}_{10}$ & $\mathbf{0 , 8 4}$ & $-0,15$ & 0,12 & $-0,11$ & 0,761 \\
\hline $\mathrm{X}_{11}$ & $\mathbf{0 , 8 0}$ & 0,18 & 0,05 & 0,31 & 0,768 \\
\hline $\mathrm{X}_{12}$ & 0,93 & 0,03 & 0,26 & 0,06 & 0,944 \\
\hline $\mathrm{X}_{13}$ & 0,23 & 0,11 & 0,93 & $-0,05$ & 0,930 \\
\hline $\mathrm{X}_{14}$ & 0,14 & 0,14 & 0,92 & $-0,06$ & 0,893 \\
\hline $\mathrm{X}_{15}$ & $\mathbf{0 , 8 0}$ & 0,04 & 0,08 & 0,07 & 0,659 \\
\hline $\mathrm{X}_{16}$ & 0,74 & 0,00 & 0,07 & 0,08 & 0,560 \\
\hline $\mathrm{X}_{17}$ & 0,92 & $-0,02$ & 0,28 & 0,03 & 0,926 \\
\hline
\end{tabular}

Fonte: Dados da pesquisa.

Nota: (*) Proporção da variância total da variável explicada pelos fatores comuns. Aparecem marcados em negrito os fatores de maior peso por variável.

Pela análise das Tabelas 1 e 2, verifica-se que o Fator 1 representa a maior parcela de variância entre os quatro fatores obtidos $(30,395 \%)$ e está associado positiva e fortemente com os indicadores $\mathrm{X}_{7}, \mathrm{X}_{10} \mathrm{X}_{11} \mathrm{X}_{12}$, $\mathrm{X}_{15}, \mathrm{X}_{16}$ e $\mathrm{X}_{17}$, que representam variáveis indicativas do nível de uso de trator, investimentos e despesas totais. O Fator 1, portanto, foi definido como "Intensidade do uso do capital".

O Fator 2 está relacionado, positiva e fortemente, com os indicadores $\mathrm{X}_{1}$ $\mathrm{X}_{2}, \mathrm{X}_{3}, \mathrm{X}_{5}$ e $\mathrm{X}_{6}$. Estas variáveis indicam práticas de uso de adubos, agroquímicos, irrigação, práticas agrícolas e acesso à energia elétrica, sugerindo um modelo mais associado ao progresso tecnológico de 
natureza química e biológica, conforme descrito por Hayami e Ruttan (1988). O Fator 2 foi definido como "Uso de tecnologia moderna".

O Fator 3, por sua vez, relaciona-se, positiva e fortemente, com os indicadores $\mathrm{X}_{9}, \mathrm{X}_{13}$ e $\mathrm{X}_{14}$, que estão associados ao valor do financiamento e ao valor bruto da produção, recebendo a denominação de "Produtividade dos fatores - terra, capital e trabalho".

O Fator 4 está associado, positiva e fortemente, com os indicadores $\mathrm{X}_{4}$ e $\mathrm{X}_{8}$, que se relacionam com o acesso aos serviços de assistência técnica e extensão rural (ATER) e financiamento, recebendo, portanto, a designação de "Cobertura de assistência técnica e financiamento".

A partir do significado de cada fator (F1= Intensidade do uso do capital; F2= Uso de tecnologia moderna; F3 = Produtividade dos fatores - terra, capital e trabalho; e F4= Cobertura de assistência técnica e financiamento) podem ser interpretados os escores obtidos na análise fatorial.

Considerando que os escores fatoriais apresentam distribuição normal, com média zero e variância unitária, pode-se interpretar que quanto maior o escore fatorial, mais avançada tecnologicamente será a agricultura do município em questão. Uma situação inversa indica atraso tecnológico, ou seja, quanto menor o escore, mais atrasada será a agricultura do município. É importante ressaltar que os escores fatoriais e a classificação obtida para cada município representam uma posição relativa diante aos demais municípios dentro da mesorregião do Nordeste Paraense, nada podendo ser inferido em relação aos demais municípios paraenses ou a outras regiões do País.

Assim, na Tabela 3, são apresentados os valores dos escores fatoriais e os indicadores bruto e relativo, na base 100, de forma que o maior valor se tornou igual a 100 e o menor igual a zero, obtendo-se uma ordenação dos municípios do Nordeste Paraense quanto ao nível tecnológico empregado na agricultura. 
São destaques na Tabela 3, quanto aos escores fatoriais, os municípios de Tailândia, Curuçá, São Miguel do Guamá e São João da Ponta, que representam os maiores valores para F1, F2, F3 e F4, respectivamente. Esses resultados mostram que Tailândia é o município da mesorregião do Nordeste Paraense com maior intensidade do uso de capital. O município de Curuçá se sobressai pelo uso de tecnologia moderna. São Miguel do Guamá destaca-se pela produtividade dos fatores terra, capital e trabalho, e São João da Ponta, pela disponibilidade de serviços de ATER e financiamento.

Os municípios de Santarém Novo, São João da Ponta e Capitão Poço foram os únicos que apresentaram valores positivos para todos os fatores, e não por acaso, estão entre os mais bem posicionados na mesorregião do Nordeste Paraense. O município de Tailândia, o mais bem ranqueado, é puxado pelo seu forte desempenho (ponto de máximo entre os 49 municípios) no Fator 1, que está associado à atuação do complexo produtivo ligado à dendeicultura, coordenado por sólido grupo empresarial. Os municípios que apresentam os escores negativos para os quatro fatores se encontram em piores situações de atraso.

Ainda com base nos escores fatoriais, foi feita a classificação destes municípios, buscando identificar de forma relativa o nível tecnológico empregado na agricultura de cada um deles. A classificação estabelecida foi determinada, comparativamente, entre o conjunto dos municípios da mesorregião estudada.

A hierarquização, por sua vez, revela que os municípios de Tailândia, Santarém Novo, São João da Ponta, Peixe-Boi e Capitão Poço se apresentam, nessa ordem, com os melhores níveis tecnológicos na agricultura. Em seguida, vêm Curuçá, São Miguel do Guamá, IgarapéAçu, Moju e Tomé-Açu, que compõem os dez municípios com melhor padrão agrícola no Nordeste Paraense (Tabela 3).

No extremo inferior da classificação, está o município de Limoeiro do Ajuru. Na sequência, vêm Colares, Oeiras do Pará, Cametá e São Domingos do Capim. 
Tabela 3 - Escores fatoriais e classificação relativa dos municípios da mesorregião do Nordeste Paraense, segundo o IBMA E IMA, 2006.

\begin{tabular}{|c|c|c|c|c|c|c|c|}
\hline Municípios & F1 & $\mathrm{F} 2$ & F3 & $\mathrm{F} 4$ & IBMA & IMA & $\begin{array}{l}\text { Posição } \\
\text { relativa }\end{array}$ \\
\hline Tailândia & 4,9224 & $-0,6752$ & 1,1546 & $-1,4870$ & 0,5618 & 100,0000 & 1 \\
\hline Santarém Novo & 0,6466 & 1,9063 & 0,5024 & 2,0097 & 0,4578 & 78,8233 & 2 \\
\hline São João da Ponta & 0,1006 & 0,9965 & 0,7129 & 4,1287 & 0,4410 & 75,3916 & 3 \\
\hline Peixe-Boi & 3,1555 & $-0,3940$ & $-1,0268$ & 0,1950 & 0,4094 & 68,9575 & 4 \\
\hline Capitão Poço & 1,1098 & 0,7044 & 0,4364 & 0,6526 & 0,3896 & 64,9250 & 5 \\
\hline Curuçá & $-0,0578$ & 2,8169 & 0,6212 & $-0,8699$ & 0,3768 & 62,3326 & 6 \\
\hline São Miguel do Guamá & $-0,9978$ & 1,3987 & 3,7524 & $-0,8624$ & 0,3636 & 59,6364 & 7 \\
\hline Igarapé-Açu & 0,3075 & 0,9478 & 0,7213 & $-0,1521$ & 0,3331 & 53,4184 & 8 \\
\hline Moju & 0,7433 & $-1,0279$ & 2,8722 & $-0,8852$ & 0,3302 & 52,8388 & 9 \\
\hline Tomé-Açu & 1,1876 & $-0,4441$ & $-0,3514$ & 1,3849 & 0,3264 & 52,0516 & 10 \\
\hline São Francisco do Pará & 0,1328 & 1,8366 & $-0,3682$ & $-0,6163$ & 0,3086 & 48,4417 & 11 \\
\hline Vigia & 0,3443 & 1,4702 & $-0,3983$ & $-0,6396$ & 0,3035 & 47,3896 & 12 \\
\hline Aurora do Pará & 0,7505 & $-1,0258$ & 0,2336 & 1,0747 & 0,2804 & 42,6941 & 13 \\
\hline Nova Esperança do Piriá & 0,0677 & $-0,2947$ & 0,7829 & $-0,0343$ & 0,2591 & 38,3457 & 14 \\
\hline Terra Alta & $-0,2811$ & 1,2559 & $-0,5145$ & $-0,1459$ & 0,2571 & 37,9469 & 15 \\
\hline Baião & $-0,5313$ & 0,1828 & 0,2021 & 1,1220 & 0,2495 & 36,4015 & 16 \\
\hline Santa Maria do Pará & $-0,2576$ & 1,7327 & $-0,6024$ & $-1,5024$ & 0,2411 & 34,6836 & 17 \\
\hline Ipixuna do Pará & 0,5263 & $-0,7653$ & $-0,7445$ & 0,9957 & 0,2361 & 33,6659 & 18 \\
\hline Tracuateua & $-0,4356$ & 1,0233 & $-0,5639$ & $-0,0712$ & 0,2344 & 33,3300 & 19 \\
\hline Bonito & $-0,0704$ & 0,4999 & $-0,5407$ & $-0,1842$ & 0,2320 & 32,8302 & 20 \\
\hline Santa & 0,3735 & 0,2105 & $-0,8$ & $-0,4994$ & 90 & 32, & 21 \\
\hline Nova Timboteua & 0,3666 & 0,1385 & $-1,1324$ & $-0,0009$ & 0,2266 & 31,7277 & 22 \\
\hline Capanema & $-0,4292$ & 0,2398 & $-0,7342$ & 1,2601 & 0,2261 & 31,6331 & 23 \\
\hline Concórdia do Pará & $-0,2843$ & $-0,5191$ & 0,3044 & 0,7139 & 0,2245 & 31,2960 & 24 \\
\hline São João de Pirabas & $-0,3727$ & $-0,6365$ & $-0,1727$ & 1,5455 & 0,2165 & 29,6807 & 25 \\
\hline Maracanã & $-0,4805$ & 0,3740 & $-0,0751$ & $-0,1948$ & 0,2146 & 29,2889 & 26 \\
\hline São Caetano de Odivelas & $-0,2881$ & 0,6288 & $-0,8687$ & $-0,0980$ & 0,2122 & 28,7956 & 27 \\
\hline Viseu & $-0,8289$ & $-0,9476$ & 2,0660 & $-0,1103$ & 0,2111 & 28,5863 & 28 \\
\hline Mocajuba & $-0,7122$ & $-0,2216$ & 0,4537 & 0,4110 & 0,2062 & 27,5830 & 29 \\
\hline Mãe do Rio & 0,3479 & $-0,4863$ & $-0,9034$ & $-0,0196$ & 0,2024 & 26,8030 & 30 \\
\hline Garrafão do Norte & $-0,3780$ & $-0,6300$ & 0,4428 & 0,1010 & 0,2004 & 26,4022 & 31 \\
\hline Irituia & $-0,1952$ & $-0,6912$ & 0,2261 & $-0,0161$ & 1985 & 26,0038 & 32 \\
\hline Ourém & 8 & -0, & $-0,0$ & -0, & 75 & 24 & 33 \\
\hline Salinópolis & $-0,4510$ & $-0,1183$ & $-1,0309$ & 0,8496 & 0,1 & 22,8663 & 34 \\
\hline Acará & $-0,3804$ & $-0,5243$ & 0,3722 & $-0,7109$ & 0,1799 & 22,2180 & 35 \\
\hline Magalhães Barata & $-0,3819$ & 0,6011 & $-0,9556$ & $-0,9519$ & 0,1765 & 21,5340 & 36 \\
\hline Marapanim & $-0,5944$ & 0,2859 & $-0,3906$ & $-0,8516$ & 0,1709 & 20,3812 & 37 \\
\hline Cachoeira do Piriá & $-0,9229$ & $-1,3858$ & 1,5235 & 0,1902 & 0,1691 & 20,0186 & 38 \\
\hline Augusto Corrêa & $-0,4013$ & $-0,0001$ & $-0,9348$ & $-0,1351$ & 0,1687 & 19,9373 & 39 \\
\hline Bragança & $-0,4398$ & 0,3543 & $-0,5297$ & $-1,2759$ & 0,1679 & 19,7708 & 40 \\
\hline Primavera & 0,1016 & $-0,4810$ & $-1,0933$ & $-0,4533$ & 0,1652 & 19,2248 & 41 \\
\hline Igarapé-Miri & $-0,8165$ & $-1,0780$ & 0,7805 & 0,2813 & 0,1650 & 19,1937 & 42 \\
\hline Quatipuru & $-0,5317$ & 0,1958 & $-0,3437$ & $-1,2483$ & 0,1615 & 18,4781 & 43 \\
\hline Abaetetuba & $-0,3777$ & $-0,9242$ & $-0,5579$ & 0,1643 & 0,1473 & 15,5906 & 44 \\
\hline São Domingos do $\mathrm{Ca}$ & $-0,3508$ & $-0,9754$ & $-0,4749$ & $-0,8019$ & 0,1228 & 10,5901 & 45 \\
\hline Cametá & $-0,5460$ & $-1,0854$ & $-0,5334$ & $-0,0786$ & 0,1213 & 10,2849 & 46 \\
\hline Oeiras do Pará & $-0,6124$ & $-1,3656$ & 0,0016 & $-0,6486$ & 0,1078 & 7,5335 & 47 \\
\hline Colares & $-0,5776$ & $-1,4532$ & $-0,7569$ & $-0,8105$ & 0,0709 & 0,0278 & 48 \\
\hline Limoeiro do A & $-0,5867$ & $-1,5776$ & $-0,7253$ & $-0,6146$ & 0,0708 & 0,0000 & 49 \\
\hline
\end{tabular}

Fonte: Dados da pesquisa. 
Fabrício Khoury Rebello, Marcos Antônio Souza dos Santos \& Alfredo Kingo Oyama Homma

O grande contraste observado entre os municípios do Nordeste Paraense reflete a necessidade de uma intervenção governamental para alavancar o desenvolvimento agrícola dos municípios mais carentes, com vistas a reduzir as disparidades intermunicipais. Isso, no entanto, não deve ser entendido como pretexto para se preterirem ações nos demais municípios, uma vez que a região como um todo apresenta um grande distanciamento dos padrões tecnológicos da média do Brasil e dos estados mais dinâmicos do País, conforme apresentado na Tabela 4.

Tabela 4 - Indicadores de modernização agrícola do Nordeste Paraense e de quatro Áreas de Referência, 2006.

\begin{tabular}{|c|c|c|c|c|c|}
\hline \multirow{3}{*}{ Indicadores } & \multicolumn{5}{|c|}{ Áreas } \\
\hline & \multirow{2}{*}{\begin{tabular}{|c|} 
Estudo \\
Nordeste \\
Paraense \\
\end{tabular}} & \multicolumn{4}{|c|}{ Referência } \\
\hline & & $\begin{array}{l}\text { Estado } \\
\text { do Pará }\end{array}$ & $\begin{array}{l}\text { Estado de } \\
\text { São Paulo }\end{array}$ & $\begin{array}{l}\text { Estado do } \\
\text { Paraná }\end{array}$ & Brasil \\
\hline $\mathrm{N}^{\circ}$ de estabelecimentos com acesso à energia elétrica (\%) & 34,16 & 27,02 & 81,43 & 77,95 & 68,14 \\
\hline $\mathrm{N}^{\circ}$ de estabelecimentos com uso de tração mecânica (\%) & 4,22 & 4,01 & 38,79 & 37,04 & 18,90 \\
\hline $\begin{array}{l}\mathrm{N}^{\circ} \text { de estabelecimentos com acesso aos serviços de } \\
\text { assistência técnica e extensão rural (\%) }\end{array}$ & 7,41 & 9,83 & 48,25 & 49,63 & 24,03 \\
\hline $\begin{array}{l}\mathrm{N}^{\circ} \text { de estabelecimentos com indicação de participação em } \\
\text { associações e cooperativas (\%) }\end{array}$ & 34,90 & 39,56 & 29,16 & 42,59 & 41,09 \\
\hline $\begin{array}{l}\mathrm{N}^{\circ} \text { de estabelecimentos com indicação de uso de práticas } \\
\text { agrícola (\%) }\end{array}$ & 22,09 & 18,63 & 54,38 & 68,83 & 44,38 \\
\hline $\begin{array}{l}\mathrm{N}^{\circ} \text { de estabelecimentos com indicação de usos de } \\
\text { queimadas (\%) }\end{array}$ & 41,27 & 33,35 & 2,04 & 2,58 & 13,56 \\
\hline $\begin{array}{l}\mathrm{N}^{\circ} \text { de estabelecimentos com indicação de uso de adubação } \\
\text { química (\%) }\end{array}$ & 19,81 & 10,27 & 50,96 & 59,50 & 32,76 \\
\hline $\begin{array}{l}\mathrm{N}^{\circ} \text { de estabelecimentos com indicação de controle de } \\
\text { pragas e doenças (\%) }\end{array}$ & 7,72 & 7,38 & 34,30 & 54,64 & 26,97 \\
\hline $\begin{array}{l}N^{\circ} \text { de estabelecimentos com indicação do uso de irrigação } \\
(\%)\end{array}$ & 2,61 & 2,16 & 12,24 & 3,41 & 6,36 \\
\hline $\begin{array}{l}\mathrm{N}^{\circ} \text { de estab elecimentos com indicação de propriedade de } \\
\text { trator (\%) }\end{array}$ & 1,91 & 2,54 & 35,16 & 20,55 & 10,25 \\
\hline $\begin{array}{l}N^{\circ} \text { de estabelecimentos com indicação de acesso a } \\
\text { financiamentos (\%) }\end{array}$ & 7,46 & 8,17 & 13,23 & 29,93 & 17,76 \\
\hline
\end{tabular}

Fonte: Elaborado pelos autores a partir de dados do Censo Agropecuário 2006 $\operatorname{IBGE}(2010)$. 
Pela análise da Tabela 4, pode-se depreender o atraso da mesorregião do Nordeste Paraense em relação às áreas de referência, que são os estados de São Paulo e Paraná, destaques nacionais em tecnologia moderna no setor rural, o estado do Pará, onde a mesorregião está inserida e o conjunto do País. É gritante o atraso quanto ao acesso à energia elétrica, financiamento, serviços de ATER, uso de tratores, controle de pragas e doenças, prática de adubação, adoção de tração mecânica, entre outros. Enquanto $7,41 \%$ das propriedades na área de estudo têm acesso aos serviços de ATER, no Brasil esse número é mais de três vezes maior ( $24,03 \%$ das propriedades). A situação piora bastante quando confrontada com a realidade do estado do Paraná, onde $49,63 \%$ das propriedades contam com esse serviço. Esse quadro se torna mais desfavorável, ainda, quando se leva em consideração o nível de capitalização dos empreendedores rurais na região Norte, principalmente os de menor porte, uma vez que eles dependem da assistência oficial prestada pelo estado que, conforme vários estudos, é crítica na Amazônia.

Em nível intermunicipal, ainda quanto ao número de propriedades atendidas pelo serviço de ATER, constata-se uma grande heterogeneidade, o que acaba por se refletir na dinâmica produtiva. Quanto a esse serviço, na melhor posição se encontra Ipixuna do Pará que tem $35,55 \%$ das propriedades rurais atendidas com algum tipo de serviço de ATER. São João da Ponta (33,33\% das propriedades), Baião (25,31\%), Peixe-Boi (23,22\%), São João de Pirabas (21,82\%) e Salinópolis $(18,04 \%)$ são os outros destaques, embora caiba a ressalva de que os municípios de São João da Ponta (39 estabelecimentos rurais em 2006), Peixe Boi (211) e Salinópolis (255) atingem esse bom grau de cobertura em função de possuírem um número bastante reduzido de estabelecimentos rurais em relação à média verificada na área de estudo (1.650 estabelecimentos). A maioria dos municípios $(63,27 \%)$ possui uma cobertura abaixo da média do estado do Pará $(9,83 \%$ das propriedades atendidas) e 42,86\% estão abaixo da média da mesorregião do Nordeste Paraense (7,41\% das propriedades atendidas). Nas piores posições, estão Oeiras do Pará (0,88\%), Limoeiro do Ajuru (1,66\%), Garrafão do Norte $(1,67 \%)$ e Colares $(1,76 \%)$, com menos de $2 \%$ das propriedades atendidas com serviços de ATER. 
Fabrício Khoury Rebello, Marcos Antônio Souza dos Santos \& Alfredo Kingo Oyama Homma

A Tabela 5 sintetiza a estratificação da mesorregião do Nordeste Paraense quanto ao nível tecnológico verificado em seus municípios para o ano de 2006.

Tabela 5 - Estratificação dos municípios do Nordeste Paraense, segundo o Índice de Modernização Agrícola (IMA), pelos três níveis tecnológicos, 2006.

\begin{tabular}{|c|c|c|c|}
\hline \multirow{2}{*}{$\begin{array}{c}\text { Níveis } \\
\text { Tecnológicos }\end{array}$} & \multicolumn{2}{|c|}{ Municípios } & \multirow{2}{*}{ Municípios } \\
\hline & Número & $\%$ & \\
\hline Nível 1 & 10 & 20,41 & $\begin{array}{l}\text { Capitão Poço, Curuçá, Igarapé -Açu, Moju, Peixe - } \\
\text { Boi, Santarém Novo, São João da Ponta, São } \\
\text { Miguel do Guamá, Tailândia, Tomé-Açu. }\end{array}$ \\
\hline Nível 2 & 22 & 44,90 & $\begin{array}{l}\text { Aurora do Pará, Baião, Bonito, Capanema, } \\
\text { Concórdia do Pará, Garra fão do Norte, Ipixuna do } \\
\text { Pará, Irituia, Mãe do Rio, Maracanã, Moc ajuba, } \\
\text { Nova Esperança do Piriá, Nova Timboteua, Santa } \\
\text { Luzia do Pará, Santa Maria do Pará, São Caetano } \\
\text { de Odivelas, São Francisco do Pará, São João de } \\
\text { Pirabas, Terra Alta, Tracuateua, Vigia, Viseu. }\end{array}$ \\
\hline Nível 3 & 17 & 34,69 & $\begin{array}{l}\text { Abaetetuba, Acará, Augusto Corrêa, Bragança, } \\
\text { Cachoeira do Piriá, Cametá, Colares, Igarapé-Miri, } \\
\text { Limoeiro do Ajuru, Magalhães Barata, Marapanim, } \\
\text { Oeiras do Pará, Ourém, Primavera, Quatipuru, } \\
\text { Salinópolis, São Domingos do Capim. }\end{array}$ \\
\hline
\end{tabular}

Fonte: Dados da pesquisa.

Percebe-se, da análise da Tabela 5, que dos 49 municípios da mesorregião do Nordeste Paraense, 20,41\% estão enquadrados no nível tecnológico 1 (IMAi > 50), o mais alto percebido na área estudada. No nível tecnológico 2(25<IMAi<50), estão 44,90\% dos municípios. Já o nível tecnológico 3 $(0<$ IMAi $<25)$, o mais baixo, contempla 34,69\% dos municípios.

Os dez municípios enquadrados no nível 1, com 15,87\% do número de estabelecimentos e $26,69 \%$ da área total da mesorregião do Nordeste Paraense, concentram $43,70 \%$ dos tratores (1.050 unidades) utilizados na atividade agrícola. Um fator determinante para esses municípios foi sua capacidade de alavancar financiamentos $(45,43 \%$ de todos os 
recursos destinados a mesorregião) e investimentos (30,23\%). O valor total das despesas desses municípios em insumos modernos representou $48,62 \%$ do total de despesas realizadas pelo conjunto dos 49 municípios estudados. A massa de recursos financeiros empregada neles, de certa forma, tem contribuído para promover externalidade positiva, com efeitos na modernização de sua agricultura, e para estimular resultados favoráveis na geração de valor bruto da produção (VBP) agropecuária, uma vez que $33,41 \%$ do VBP gerado no Nordeste Paraense se concentra nesses 10 municípios, com destaques para as lavouras permanentes $(35,86 \%)$, lavouras temporárias $(37,62 \%)$, silvicultura $(41,96 \%)$, animal $(46,79 \%)$ e aves $(60,48 \%)$.

Os resultados obtidos apontam para a necessidade de se trabalhar, de forma mais intensa, na elevação do nível tecnológico nessa mesorregião, no sentido de melhorar seu dinamismo econômico e, por conseguinte, o nível de vida no campo.

4.2. Mudança na estrutura da modernização da agricultura entre os anos de 1996 e 2006

Rebello, Santos e Homma (2010), adotando a mesma metodologia empregada neste trabalho, identificaram o nível tecnológico para os municípios do Nordeste Paraense, considerando o ano de 1995/96, conforme apresentado na Tabela 6.

Dos 45 municípios existentes na mesorregião do Nordeste Paraense, considerando o ano de 1995/1996, 8,89\% estavam enquadrados no nível tecnológico 1 (IMAi>50), o mais alto percebido na área estudada. O nível tecnológico $2(25<\mathrm{IMAi}<50)$, contemplava $17,78 \%$ dos municípios. Já no nível tecnológico $3(0<\mathrm{IMAi}<25)$, o mais baixo, estavam 73,33\% dos municípios. 
Fabrício Khoury Rebello, Marcos Antônio Souza dos Santos \& Alfredo Kingo Oyama Homma

Tabela 6 - Estratificação dos municípios do Nordeste Paraense, segundo o Índice de Modernização Agrícola (IMA), pelos três níveis tecnológicos, 1995/1996.

\begin{tabular}{c|c|c|l}
\hline \multirow{2}{*}{$\begin{array}{c}\text { Níveis } \\
\text { Necnológicos }\end{array}$} & \multicolumn{2}{|c|}{ Municípios } & \multicolumn{1}{c}{ Municípios } \\
\cline { 2 - 3 } Nível 2 & 4 & 8,89 & Curuçá, Igarapé-Açu, São Francisco do Pará, Tailândia. \\
\hline \multirow{2}{*}{ Nível 3 } & 8 & 17,78 & $\begin{array}{l}\text { Ipixuna do Pará, Mãe do Rio, Nova Timboteua, Peixe -Boi, } \\
\text { Santarém Novo, São Caetano de Odivelas, Terra Alta, } \\
\text { Vigia. }\end{array}$ \\
\hline & 33 & 73,33 & $\begin{array}{l}\text { Abaetetuba, Acará, Augusto Corrêa, Aurora do Pará, Baião, } \\
\text { Bonito, Bragança, Cametá, Capanema, Capitão Poço }, \\
\text { Colares, Concórdia do Pará, Garrafão do Norte, Igarapé - } \\
\text { Miri, Irituia, Limoeiro do Ajuru, Magalhães Barata, } \\
\text { Maracanã, Marapanim, Mocajuba, Moju, Nova Esperança } \\
\text { do Piriá, Oeiras do Pará, Ourém, Primavera, Salinópolis, } \\
\text { Santa Luzia do Pará, Santa Maria do Pará, São Domingos } \\
\text { do Capim, São João de Pirabas, São Miguel do Guamá, } \\
\text { Tomé-Açu,Viseu. }\end{array}$ \\
\hline
\end{tabular}

Fonte: Rebello, Santos e Homma (2010).

Nota: Em 1995/1996, os municípios de Cachoeira do Piriá, Quatipuru, São João da Ponta e Tracuateua não haviam sido emancipados, portanto, as análises foram desenvolvidas considerando 45 municípios e não os 49 atualmente existentes.

Comparando essa situação com a realidade de 2006, percebe-se uma evolução positiva, pois o nível tecnológico 1 passou a contar com 10 municípios, ao invés de 4. O nível 2, com 22 municípios (contra 8, em 1995/1996), e o nível tecnológico 3, que era o mais representativo em 1995/1996, com 33 municípios (73,33\% do universo), sofre uma redução significativa, contando, em 2006, com 17 municípios (34,69\% dos municípios).

Muitos municípios mudaram de posição relativa entre os dois períodos analisados. São Francisco do Pará caiu do nível tecnológico 1 para o 2 . Capitão Poço, Moju, São Miguel do Guamá e Tomé-Açu saíram do nível tecnológico 3 para ingressar no 1. Peixe Boi e Santarém Novo, por sua vez, passaram do nível 2 para o 1 . Quinze municípios ascenderam do nível tecnológico 3 para o 2 (Aurora do Pará, Baião, Bonito, Capanema, 
Concórdia do Pará, Garrafão do Norte, Irituia, Maracanã, Marapanim, Mocajuba, Nova Esperança do Piriá, Santa Luzia do Pará, Santa Maria do Pará, São João de Pirabas e Viseu). Quatorze mantiveram sua posição no nível 3 (Abaetetuba, Acará, Augusto Corrêa, Bragança, Cametá, Colares, Igarapé-Miri, Limoeiro do Ajuru, Magalhães Barata, Oeiras do Pará, Ourém, Primavera, Salinópolis e São Domingos do Capim). Dos quatro novos municípios emancipados, somente São João da Ponta ingressou no nível 1. Tracuateua ficou no nível 2. Cachoeira do Piria e Quatipuru, no nível tecnológico 3.

\section{Considerações finais}

O estudo revelou a existência de considerável discrepância no nível tecnológico entre os municípios da mesorregião do Nordeste Paraense, evidenciando um contraste nos indicadores relacionados à modernização da agricultura na área estudada, ainda que tenha sido percebida significativa evolução entre os períodos de 1995/1996 e 2006. Percebeuse, também, um distanciamento desfavorável destes municípios em relação aos padrões vigentes na média do Brasil e aos estados mais dinâmicos, principalmente quanto ao acesso à energia elétrica, uso de tração mecânica, acesso aos serviços de assistência técnica e ao crédito e o uso de práticas agrícolas modernas.

Dos 49 municípios analisados, considerando o ano de 2006, dez atingiram índice de modernização agrícola acima de 50\%, quando comparados entre si. São eles, na ordem, Tailândia, Santarém Novo, São João da Ponta, Peixe-Boi, Capitão Poço, Curuçá, São Miguel do Guamá, Igarapé-Açu, Moju e Tomé-Açu.

O estudo também evidenciou certa mobilidade dos municípios no sentido de atingir patamares mais elevados de tecnologia considerando os últimos dez anos (1995/1996 a 2006). Em 1995/1996, 73,33\% dos municípios da mesorregião do Nordeste Paraense foram enquadrados no nível tecnológico 3, o mais atrasado. Em 2006, esse nível tecnológico passa a 
Fabrício Khoury Rebello, Marcos Antônio Souza dos Santos \& Alfredo Kingo Oyama Homma

ser representado por $34,69 \%$ dos municípios, ou seja, quase a metade ascendeu a patamares mais elevados de tecnologia.

Essa mobilidade para níveis mais elevados de tecnologia, em boa parte, está associada ao cultivo de culturas empresariais como o dendê, pimentado-reino, laranja, maracujá, coco-da-baía, que têm exigido a adoção de níveis tecnológicos diferenciados e, assim, criado uma externalidade positiva ao conjunto das atividades agrícolas no município.

Considerando que uma das alternativas mais eficientes para se evitar a ocupação desenfreada da floresta amazônica é o estímulo ao aumento da produtividade agrícola e pecuária, em áreas mais vocacionadas para essas finalidades, conforme recomendado por Rebello e Homma (2009), entende-se que a elevação dos níveis tecnológicos nessa mesorregião possa ser uma ação estratégica relevante. Assim, deve-se dar atenção redobrada às políticas públicas relacionadas à modernização da agricultura na Amazônia, principalmente na área de estudo, por considerar seu nível de antropização e de importância relativa no contexto econômico e social.

\section{Referências}

\section{BILLOT, A. Agriculture et systèmes d'élevage en zone Bragantine} (Pará-Brasil) : diagnostique des systèmes de production familiaux à forte composante élevage. 1995. 140 f. Master (Thesis) - Centre National d'Études Agronomiques des Régions Chaudes, Montpellier, França.

CUNHA, N. R. S.; LIMA, J. E.; GOMES, M. F. M.; BRAGA, M. J. A intensidade da exploração agropecuária como indicador da degradação ambiental na região dos Cerrados, Brasil. Revista de Economia Rural, Piracicaba, SP, v.46, n.2, p. 291-323, abr./jun. 2008.

CUNHA, N. R. S.; LIMA, J. E.; MOURA, L. R. C. Degradação ambiental nos Estados de Goiás e Tocantins. In: CONGRESSO BRASILEIRO DE ECONOMIA E SOCIOLOGIA RURAL, 43, 2005, 
Ribeirão Preto, SP. Anais... Ribeirão Preto, SP: SOBER, 2005. CDROM.

EGLER, E. G. A. Zona Bragantina no Estado do Pará. Revista Brasileira de Geografia, Rio de Janeiro, v. 23, p. 527-555, 1961.

HAIR, J. F.; ANDERSON, R. E.; TATHAN, R. L.; BLACK, W. C. Análise multivariada de dados. $5^{\mathrm{a}}$ ed. Porto Alegre: Bookman, 2006.

HAYAMI, Y.; RUTTAN, V. Desenvolvimento agrícola: teoria e experiências internacionais. Brasília, DF: Embrapa, 1988.

HOMMA, A. K. O. Amazônia: meio ambiente e desenvolvimento agrícola. Brasília, DF: EMBRAPA, SPI, 1998.

INSTITUTO BRASILEIRO DE GEOGRAFIA E ESTATÍSTICA. Censo agropecuário 1995/96. Rio de Janeiro, 1998. Disponível em: $<$ http://www.ibge.gov.br/servidor_arquivos_est/>. Acesso em: $17 \mathrm{fev}$. 2010.

Censo agropecuário 2006. Rio de Janeiro, 2010. Disponível em: $<$ http:// www.ibge.gov.br / servidor_arquivos_est $/>$. Acesso em: 15 jun. 2010.

. Censo demográfico 2000. Disponível em: <http:// www.sidra.ibge.gov.br>. Acesso em: 17 maio 2009.

.Pesquisa agrícola municipal. Disponível em: $<$ http:// www.ibge.gov.br>. Acesso em: 25 nov. 2010a.

MANLY, B. F. J. Métodos estatísticos multivariados: uma introdução. $3^{\mathrm{a}}$ ed. Porto Alegre: Bookman, 2008.

MELO, C. O.; PARRÉ, J. L. Caracterização do desenvolvimento rural dos municípios paranaenses. In: CONGRESSO BRASILEIRO DE ECONOMIA E SOCIOLOGIA RURAL, 44, 2006, Fortaleza. Anais... Fortaleza: SOBER, 2006. CD-ROM. 
Fabrício Khoury Rebello, Marcos Antônio Souza dos Santos \& Alfredo Kingo Oyama Homma

MINGOTI, S. A. Análise de dados através de métodos de estatística multivariada: uma abordagem aplicada. Belo Horizonte: EDUFMG, 2005.

PAIVA, R. M. Modernização e dualismo tecnológico na agricultura: uma reformulação. Pesquisa e Planejamento Econômico, Rio de Janeiro, v. 5, n. 1, p. 117-161, jun. 1975.

PENNA, J.A.; MUELLER, C. C. Fronteira agrícola, tecnologia e margem intensiva: algumas reflexões sobre o papel desses fatores para o crescimento agrícola brasileiro. Estudos econômicos, São Paulo, v. 7, n. 1, p. 53-106, jan./abr. 1977.

PENTEADO, A. R. Problemas de colonização e de uso da terra na Região Bragantina do Estado do Pará. Belém: UFPA, 1967.

REBELLO, F. K.; HOMMA, A. K. O. Estratégias para reduzir desmatamentos e queimadas na Amazônia. In: VEIGA, J. E. (Org.). Economia socioambiental. São Paulo: Senac, 2009.

REBELLO, F. K.; SANTOS, M. A. S.; HOMMA, A. K. O. Modernização da agricultura na mesorregião do Nordeste Paraense (PA): determinantes e hierarquização em 1996. Movendo Idéias, Belém, v. 15, n. 1, 2010. 
REVISTA DE ECONOMIA E AGRONEGÓCIO, VOL.9, $N^{\circ} 2$ 